\title{
OBSERVATION OF THE DYNAMIC BETA EFFECT FOR VARIOUS BUNCH LENGTHS AT VEPP-2M.
}

\author{
P.M. Ivanov, I.N. Nesterenko*, A.A. Valishev, \\ Budker Institute of Nuclear Physics, 630090, Novosibirsk, Russia
}

\begin{abstract}
For particles with small betatron amplitudes the beambeam interaction is like additional linear focusing which results in the beta function distortion. In this paper the observations of this so-called dynamic beta effect for various bunch lengths at the VEPP-2M collider are presented.
\end{abstract}

\section{INTRODUCTION}

In the first approximation the beam-beam interaction can be considered as an additional focusing lens which perturbs the initial lattice functions of a storage ring. The distortion of the lattice function results in reduction of the beam life time and essentially influences the background loading of the detector. The influence of dynamic beta effect on CESR operation has initiated a series of papers $[1,2]$, in which the feasible methods of this effect reduction are discussed. Distortion of the storage ring lattice functions leads to the change in generation conditions of the beam emittance. Therefore it is necessary to take this effect into consideration in simulation of the beam-beam interaction and provide the dynamic perturbation of the emittance in the presence of counter-beam as well as the correct generation of its initial value. The paper [3] gives the analytical calculation of this effect.

In this work the experimental results of the dynamic beta effect observation with different values of the bunch length $\sigma_{s}$ and the $\beta$-function at IP $\beta^{*}$ are presented. They were received at the VEPP-2M collider (Novosibirsk) [4] in summer, 2000. A computer simulation of this effect for various values of the parameter $\sigma_{s} / \beta^{*}$ has been carried out and the results have been compared to the experiment.

\section{BASIC FORMULAE}

For particles with small betatron oscillation amplitudes ( $<\sigma, \sigma$ being the Gaussian beam size) the opposite bunch can be considered as a focusing lens with the gradient which depends only on longitudinal coordinate $s$ :

$$
g_{x, y}(s)=g_{x, y}^{*} \cdot \exp \left(-\frac{s^{2}}{2 \sigma_{e f f}^{2}}\right) / \sqrt{1+\frac{s^{2}}{\beta_{x, y}^{* 2}}} .
$$

Here $\sigma_{\text {eff }}=\sigma_{s} / 2$ is the effective length of the beambeam interaction region. Exponential dependence results from the Gaussian distribution of particles in a bunch and

\footnotetext{
*nesterenko@inp.nsk.su
}

the term $\sqrt{1+s^{2} / \beta_{x, y}^{* 2}}$ is related to the change of verti$\mathrm{cal} /$ horizontal beam size caused by the $\beta$-function variation in experimental straight section. Coefficient $g_{x, y}^{*}$ is derived from the normalizing condition:

$$
\oint \beta_{x, y}(s) \cdot g_{x, y}(s) d s=4 \pi \xi_{x, y},
$$

$\xi_{x, y}$ is the beam-beam space charge parameter.

Calculation of the $\beta$-function distortion in the storage ring VEPP-2M has been done using the code RING. The beam was sliced in longitudinal direction in 30 parts (lenses) with equal length. The gradient value in each lens varied vs. its position relative to the IP according to the formula (1). The $4 \times 4$ matrix which transforms the betatron coordinates through a slice has block-diagonal structure:

$$
\left(\begin{array}{cc}
G_{x} & 0 \\
0 & G_{y}
\end{array}\right)
$$

Here $G_{x, y}$ are $2 \times 2$ matrices of the focusing quadrupole lens.

\section{EXPERIMENTAL RESULTS}

The vertical beam size was measured using digital cameras with linear CCD-sensor, one for the positron bunch and two for the electron bunch. One of the electron cameras was located in a place symmetric to the positron camera (with equal the $\beta$-function values and the betatron phase with respect to IP). The betatron phase between the observation and interaction points equals to $\pi / 2$. Another electron camera was located in an additional place with the betatron phase difference with respect to IP equal to $\pi$ (Fig.1). If the beam size is measured in two proper points (with the betatron phase difference of $\pi / 2$ ) then it is possible to receive information about the beam-beam space charge parameter $\xi$ value without any external influence on the beam.

The collider was operated at the beam energy E=392 $\mathrm{MeV}$ in strong-weak operation mode. The positron beam always had greater current. Such regime eliminates possible additional focusing of electrons due to the presence of ion clouds. The electron beam current was considered small if the relative change of the strong positron beam size was less than $10 \%$.

We have chosen the following ratio as the value describing dynamic beta effect:

$$
\frac{\tilde{\sigma}_{3 A}^{2}}{\tilde{\sigma}_{4 A}^{2}} / \frac{\sigma_{3 A}^{2}}{\sigma_{4 A}^{2}}=\frac{\tilde{\beta}_{3 A}}{\tilde{\beta}_{4 A}} / \frac{\beta_{3 A}}{\beta_{4 A}} .
$$




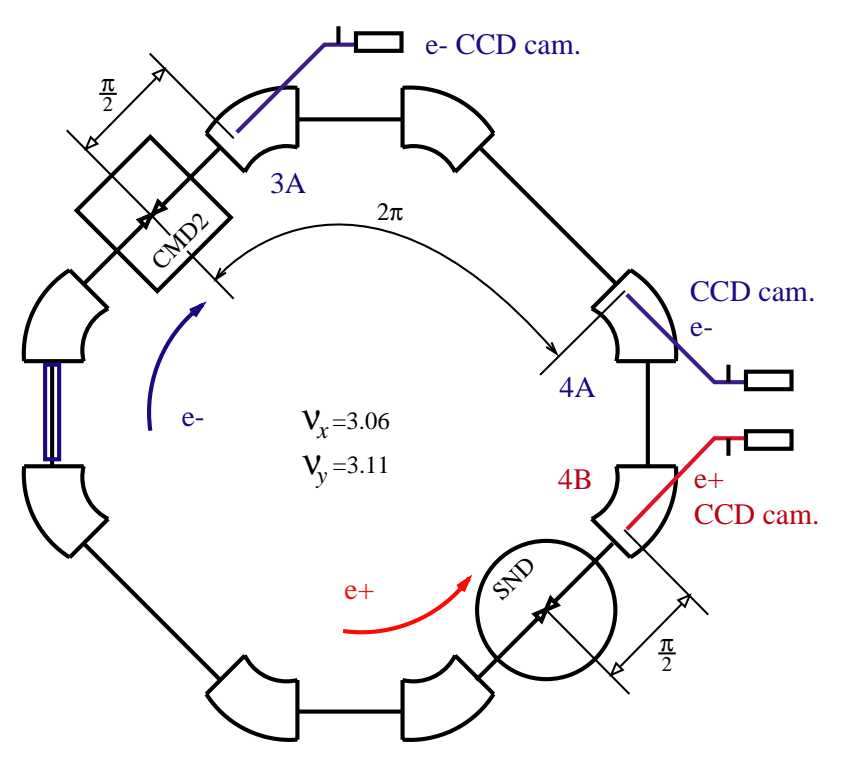

Figure 1: Layout of the experiment.

Here $\sigma_{3 A}, \sigma_{4 A}, \beta_{3 A}, \beta_{4 A}$ are the electron beam sizes and beta-functions in the absence of positron beam at observation points 3A, 4A, accordingly, and $\tilde{\sigma}_{3 A}, \tilde{\sigma}_{4 A}, \tilde{\beta}_{3 A}, \tilde{\beta}_{4 A}$ are these values with the presence of the positron beam. This ratio does not depend any more on the beam emittance and can describe the "clean" dynamic beta effect. Normalization on $\sigma_{3 A}^{2} / \sigma_{4 A}^{2}$ is done for equal scaling of all results. In Fig. 2 the normalized beam sizes vs. the positron beam current are shown. The beam size at the $4 \mathrm{~A}$ observation point grows regardless of the fact that $\delta \beta_{4 A}$ (distortion of the $\beta$-function) is negative. This fact shows that the beam emittance growth is much more than dynamic distortion of the $\beta$-function at this point.

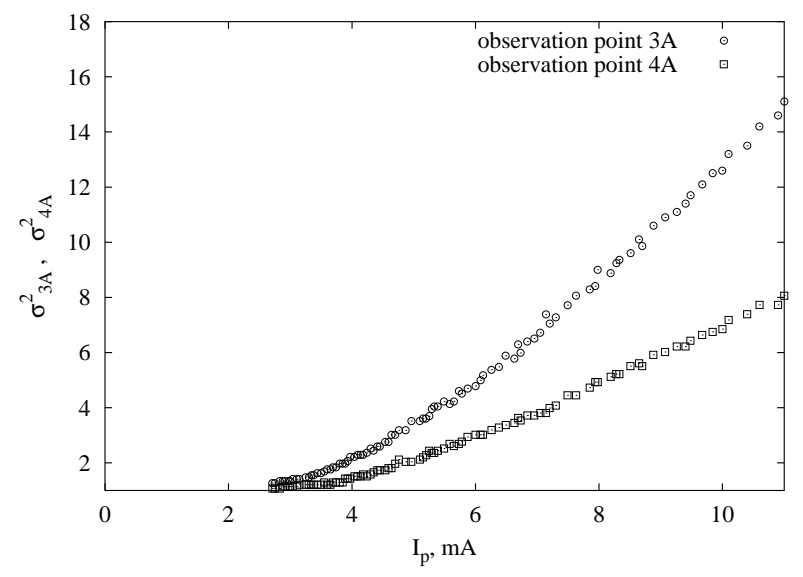

Figure 2: Normalized sizes of the electron beam at two observation points vs. the positron beam current.

Ratio $\sigma_{s} / \beta^{*}$ is the key parameter influencing the dynamic distortion of the storage ring lattice functions. It is possible to provide various values of this parameter in two ways:
- by controlling the accelerating RF field voltage

- by changing the $\beta^{*}$ value.

We used both ways of this parameter variation to expand the range of its accessible values.

In Figs. 3,4,5,6 the experimental data and the numerical calculation results of the dynamic beta effect for some typical values of parameter $\sigma_{s} / \beta^{*}$ and two different values of the $\beta$-function at IP $\left(\beta^{*}=4.2 \mathrm{~cm}\right.$ and $\left.\beta^{*}=4.8 \mathrm{~cm}\right)$ are demonstrated. Satisfactory agreement of the simulation results with received experimental data is observed. Visible deviations of the simulation from the experiment in Fig.3 at high $\xi$ values are apparently connected with the nonlinearity of the beam-beam interaction which is not taken into account in simulation. These deviations demand additional detailed research.

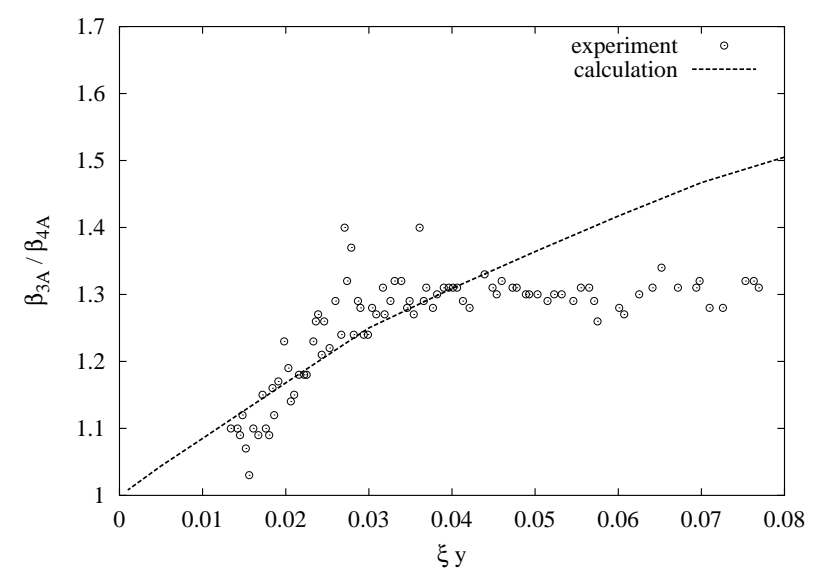

Figure 3: Ratio of the dynamic $\beta$-functions at two observation points (3A and $4 \mathrm{~A}$ in Fig.1) vs. the beam-beam parameter $\xi$. Initial $\beta_{3 A} / \beta_{4 A}=2.0$, ratio of the longitudinal beam size and beta-function at the IP $\left(\sigma_{s} / \beta^{*}\right)$ was 1.2. Relative beta distortion at the $\operatorname{IP}\left(\delta \beta^{*} / \beta^{*}\right)$ at $\xi=0.08$ was 0.31 .

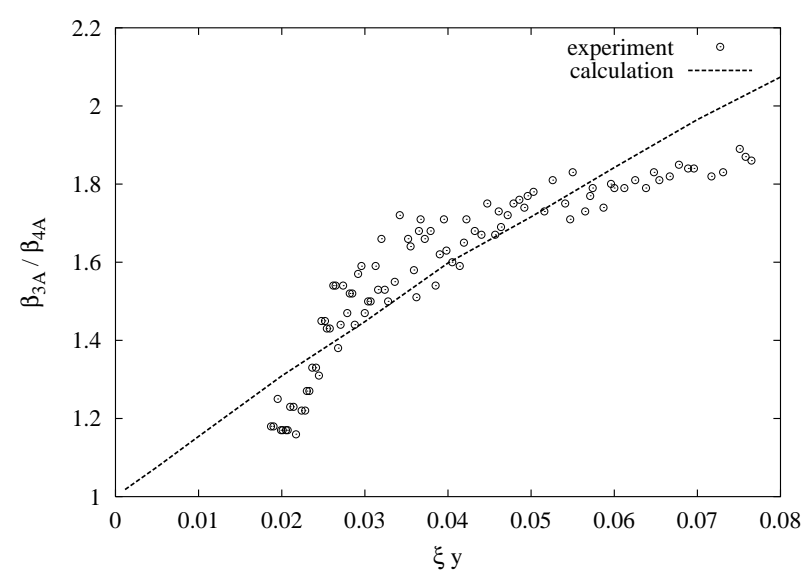

Figure 4: Ratio of the dynamic $\beta$-functions at two observation points vs. the beam-beam parameter $\xi$. Initial $\beta_{3 A} / \beta_{4 A}=2.0, \sigma_{s} / \beta^{*}=0.5, \delta \beta^{*} / \beta^{*}=0.36$. 


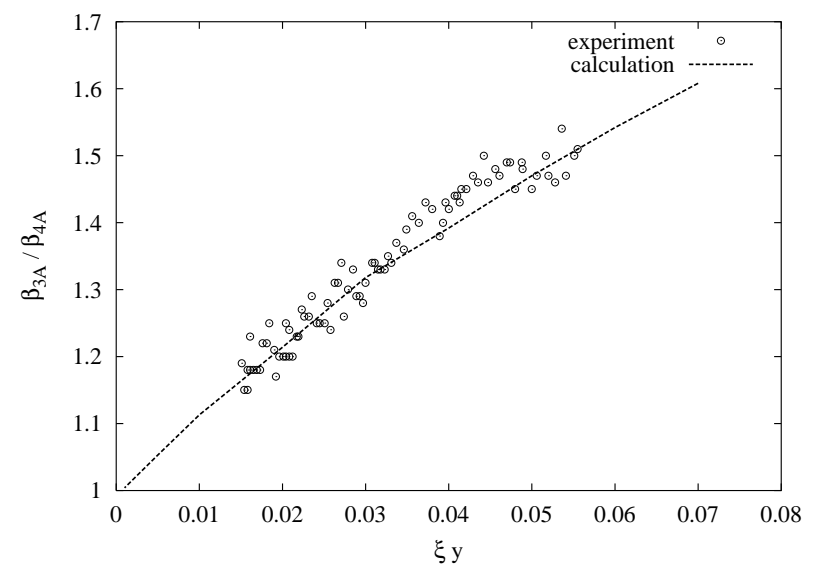

Figure 5: Ratio of the dynamic $\beta$-functions at two observation points vs. the beam-beam parameter $\xi$. Initial $\beta_{3 A} / \beta_{4 A}=1.6, \sigma_{s} / \beta^{*}=1.0, \delta \beta^{*} / \beta^{*}=0.29$.

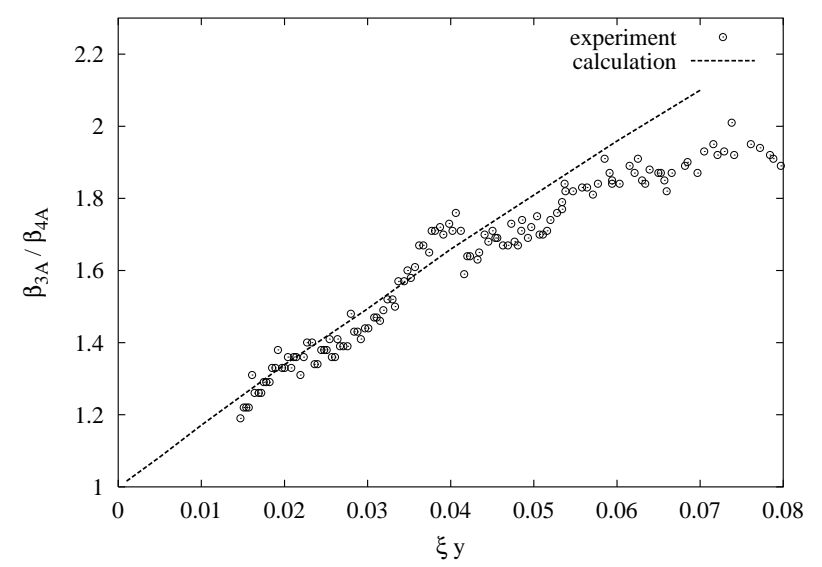

Figure 6: Ratio of the dynamic $\beta$-functions at two observation points vs. the beam-beam parameter $\xi$. Initial $\beta_{3 A} / \beta_{4 A}=1.6, \sigma_{s} / \beta^{*}=0.4, \delta \beta^{*} / \beta^{*}=0.35$.

\section{CONCLUSION}

Preliminary results of the experimental data treatment and simulation of the dynamic beta effect show satisfactory agreement. Distinctions revealed in the experimental dependencies of the dynamic beta effect require further interpretation. In the future it is planned to compare the experimental data with results of strong-weak beam-beam simulation codes.

\section{ACKNOWLEDGMENTS}

We thank E.A. Perevedentsev and A.V. Otboyev for helpful discussions during preparation of the paper. We are grateful to Yu.M. Shatunov for his support and help in the experiments.

\section{REFERENCES}

[1] D. Sagan, "The Dynamic Beta Effect in CESR", CBN 94-06, Cornell 1994.

[2] A. Temnykh, "The Dependence of Dynamic Beta Effect on Bunch Lenght”, CON 96-14, Cornell, June 1996.

[3] A.V. Otboyev, E.A. Perevedentsev, "Self-consistent beta function and emittances of round colliding beams", PRST$\mathrm{AB}$, vol.2, 104401, 1999.

[4] P.M. Ivanov, I.A. Koop, E.A. Perevedentsev, Yu.M. Shatunov and I.B. Vasserman, "Luminosity and the beam-beam effects in the electron-positron storage ring VEPP-2M with superconducting wiggler magnet", in Proc. 3rd Advanced ICFA Beam Dyn. Workshop, May 29 - June 3, Novosibirsk, 1989, p. 26 (1989). 